\title{
Dual-Operability and Bandwidth Partitioning Enabled by an ONU With Tandem-Modulator
}

\author{
Bernhard Schrenk, Johan Bauwelinck, Jose A. Lazaro, Christophe Kazmierski, Xing-Zhi Qiu, and \\ Josep Prat
}

\begin{abstract}
An upgrade at the physical layer regarding unbundling and dynamic bandwidth provisioning of a wavelength division multiplexed access network is presented. The capability of a reflective modulator to act as a photodetector is exploited and allows a tandem configuration to be used at the optical network unit (ONU) for flexible switching of the detection and modulation branch. Consequently, a mechanism for service operator selection and for dynamic bandwidth partitioning up to twice the nominal data rate is provided. The scheme is experimentally evaluated for different kinds of modulators and shows, together with a full-duplex transmission at $10 \mathrm{~Gb} / \mathrm{s}$, additional operation modes, featuring unidirectional downstream and upstream burst-mode transmission with up to $20 \mathrm{~Gb} / \mathrm{s}$ for ONUs based on a combination of a semiconductor optical amplifier and a reflective electro-absorption modulator.
\end{abstract}

Index Terms-Dynamic bandwidth allocation; Multioperability; Optical communication terminals; Optical fiber communication; Optical signal detection; Passive optical network.

\section{INTRODUCTION}

$\mathbf{I}$ mmense and widespread deployment of multimedia services can already be foreseen in the near future [1]. The maturity of the devices used for optical telecommunication and the extensive research that was carried out in recent years predicts commercial applications for the access segment $[2,3]$. Reasonably low expenditures can thereby be achieved with passive optical networks (PONs), which are free of electrically powered equipment in the fiber plant. The cost efficiency can be further improved by a shared infrastructure. For this reason, multiplexing techniques are typically incorporated in the network topology. In particular, wavelength division

Manuscript received March 3, 2011; accepted July 4, 2011; published August 18, 2011 (Doc. ID 143584).

Bernhard Schrenk (e-mail: schrenk@mail.ntua.gr) was with the Technical University of Catalonia, Barcelona, Spain, and is now with the School of Electrical and Computer Engineering, National Technical University of Athens, 15773 Athens, Greece.

Johan Bauwelinck and Xing-Zhi Qiu are with the Department of Information Technology (IMEC/INTEC), Ghent University, 9000 Ghent, Belgium.

Jose A. Lazaro and Josep Prat are with the Department of Signal Theory and Communication, Technical University of Catalonia, 08034 Barcelona, Spain.

Christophe Kazmierski is with the Alcatel-Thales III-V Lab, a joint laboratory of Alcatel-Lucent Bell Labs France and Thales Research \& Technology, Marcoussis 91461, France.

Digital Object Identifier 10.1364/JOCN.3.000674 multiplexing (WDM) provides a smooth upgrade for the PON since the introduced signal distribution element typically has low insertion losses [4,5]. In turn, a virtual point-to-point connectivity is created by the WDM overlay, inferring isolated data channels dedicated to the particular network users. If the customer premises equipment is left without wavelengthspecific elements, a possible mass deployment of the colorless optical network unit (ONU) without inventory management problems guarantees the lowest capital expenditures.

Impressive strides have recently been made in research; data transmission in such carrier-distributed WDM-PONs with separate wavelengths for downstream and upstream has already been demonstrated at data rates of up to $10 \mathrm{~Gb} / \mathrm{s}$ with colorless transmitters or reflective modulators at the ONU [6,7]. The data rate can be further increased by using several pairs of receivers and transmitters at the ONU [8].

However, the bursty nature of data traffic and the time-varying data rates of downstream and upstream can impose a limit for the data throughput on the application layer. The content delivery of video, for example, favors either highly asymmetrical data rates in the case of broadcasting or symmetrical rates for peer-to-peer streaming [9]. Consequently, advanced functionalities for steering the burst-like delivery of huge data contents become necessary to guarantee smooth data transmission for all users of the shared network. The mechanisms used to do so are often found at the higher layers. Alternatively, providing this added functionality at the physical layer can be, as we will show in this paper, beneficial if no significant cost and performance limitations arise.

Although approaches for dynamic bandwidth partitioning already exist, they mostly rely on a fixed physical design for the ONU and guarantee provisioning in the course of the delivered data rates in time division multiplexed networks, where the data rate per network segment is shared among a bunch of users [10]. Similarly, such an allocation scheme is also possible for dense WDM-PONs with a reduced set of optical transmitters [11]. As an attractive alternative, orthogonal frequency division multiplexing provides a flexible way to implement a dynamic bandwidth allocation mechanism [12,13]; however, the required capabilities for electronic real-time signal processing at high data rates often impose a limit for applicability due to energy-hungry electronic subsystems [14].

On top of dynamic bandwidth allocation, regulator bodies ask for unbundling and multi-operability, where services are delivered by two or more operators that share the same 

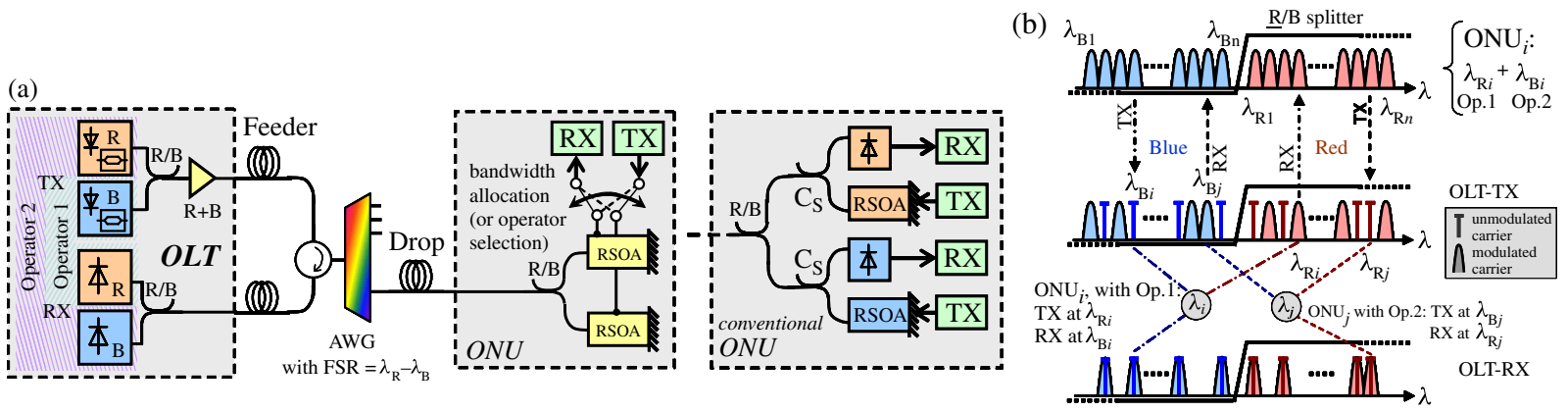

Fig. 1. (Color online) (a) Dual-operated PON with dynamic bandwidth partitioning. A pair of wavelengths is fed via the free spectral range property of the AWG toward the ONU that is designed with an identical detector and remodulator. The alternative ONU, which duplicates its receivers and transmitters, is shown on the right. (b) Wavelength allocation to support dual-operability and dynamic bandwidth partitioning for $n$ different ONUs fed by the wavelengths $\lambda_{\mathrm{R} 1}+\lambda_{\mathrm{B} 1}$ to $\lambda_{\mathrm{Rn}}+\lambda_{\mathrm{Bn}}$.

infrastructure [15]. This extension can also be made at the physical layer if the fiber plant itself is not amenable to unbundling, by dedicating separate wavelengths for the operators-presuming a balanced number of customers to stay efficient. The selection of the preferred operator is then in principle possible by inserting an optical filter at the ONU; however, this may not be seen as transparent enough for the user.

In this work we present a method for dual-operability and dynamic bandwidth provisioning in a carrier-distributed WDM-PON. We show that if a reflective modulator is used as a detector [16,17], a way for switching the operator can be provided with a tandem-modulator configuration at the ONU. At the same time, the limitations for the maximum unidirectional data rate, which derive from the electro-optical bandwidth of a single detector (or modulator) at the ONU, are overcome and a mechanism for bandwidth allocation can be exploited without the need for extensive signal processing in the electrical domain [12,13].

Experimental validation of the proposed concepts are given for an ONU that is based on a reflective semiconductor optical amplifier (RSOA), as well as for an integrated semiconductor optical amplifier/reflective electro-absorption modulator (SOA/REAM), as was recently demonstrated in brief $[18,19]$, however, without detailed treatment.

\section{INTEGRATION OF HigheR LAYER Functionalities at the Physical Layer}

The key to transferring basic functionality from the higher to the physical layer is a symmetrical ONU design that employs a device which is capable of performing two functions (Fig. 1(a)): detection of the downstream and remodulation of an optical carrier with upstream data. In both branches of the ONU, each one dedicated to one of two wavelengths as in [6], a reflective modulator performs the desired task of either detection or modulation. These basic functionalities have been particularly demonstrated for RSOA and SOA/REAM devices [16,20]. A tandem configuration of such components inside the ONU permits either dual-operability or dynamic bandwidth partitioning functionalities in broadband access networks, as will now be explained.

\section{A. Dual-Operability}

According to the first aspect of this work, namely, the dual-operability of the access network, the two delivered wavelengths for each ONU are assigned to the two operators potentially sharing the PON infrastructure. The allocation of the optical carriers, illustrated in Fig. 1(b), is chosen so that the downstream of these operators is transmitted in the red and the blue waveband. Since a red/blue filter (R/B) acts as the separator of the detection and modulation branch inside the ONU, the two downstream signals of the particular service operators will arrive at the different reflective modulators. Depending on which of them is biased for detection, it is possible to select the operator (i.e., the appropriate wavelength) by electrical means.

Subsequently, we specify the upstream operation. Since each ONU is fed by two wavelengths but just a single downstream, the wavelength that is assigned to the second operator that is actually not chosen for downstream transmission can be left unmodulated. At the ONU, this optical seed at the opposite waveband than the received downstream carrier, arrives at the second reflective modulator that can be operated as an upstream transmitter. Accordingly, the receivers at the optical line terminal (OLT) for both operators will have the reverse wavelength allocation than the OLT transmitters. Thus, a maximum use of the available optical spectrum is obtained, as optical carriers non-operative for the downstream are used for the upstream, and dual-operability does not imply halving the optical carrier availability of the WDM-PON.

An example for this scheme follows. In the case of ONU $i$ that would receive the $i$ th pair of wavelengths at $\lambda_{\mathrm{Bi}}$ and $\lambda_{\mathrm{Ri}}$, the user has chosen operator 1 corresponding to Fig. 1(b). As shown in Fig. 2(a), the inactive wavelength allocated to the operator (e.g., $\lambda_{\mathrm{Bi}}$ in the blue waveband) is transmitted unmodulated at the OLT transmitter to allow the user to imprint it with upstream toward operator 1 , which awaits this signal (at $\lambda_{\mathrm{Bi}}$ ) at the OLT receiver.

Service operators can now be switched electrically by simply choosing the appropriate bias current of the reflective modulator while reversing the direction of the $\mathrm{RF}$ amplifier. 


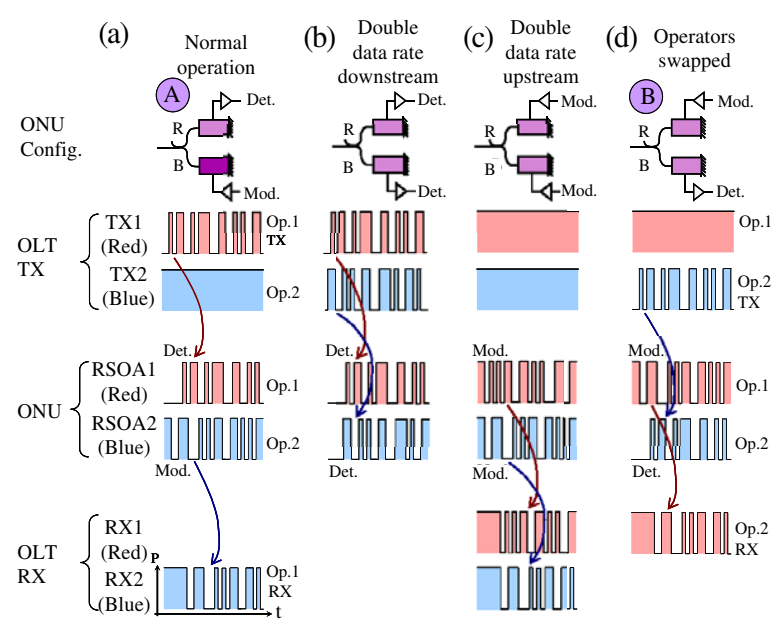

(e)

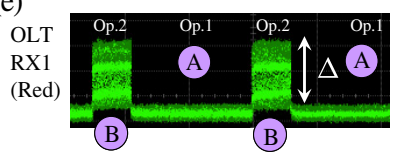

Fig. 2. (Color online) (a)-(d) Different modes of operation for the ONU regarding dynamic bandwidth allocation or a switch of the service operators. (e) Received optical signals at one of the OLT receivers in the case of switching the operator at the ONU.

In this way the dedication between downstream and upstream branches (i.e., the wavebands for the reflective modulator) is swapped and operator 2 is selected, as is sketched in Fig. 2(d).

Note that for the switch between operators simple signaling information is required for the OLT to change the dedication of the downstream transmitters. Although this can be done at the higher layers by implementing a simple protocol, the information can be in principle retrieved by the OLT just by monitoring the received optical signals. Since one upstream receiver is left unused (e.g., $\mathrm{RX} 1$ at $\lambda_{\mathrm{Ri}}$ in normal operation) as it does not receive information but an extinct optical signal as long as the ONU drops the optical signal to its electrical detector [20], this detector will notice a change of the operator due to the presence of upstream data once this switch is made by the ONU. Figure 2(e) shows the received upstream signals for RX1 at the OLT in the case of such an operator change. As can be seen, by monitoring the relative difference $\Delta$ in the received optical signal, which is up to $30 \mathrm{~dB}$ [20], a switch of the operator can be deduced and the transmitters of the OLT reconfigured as described earlier. As a partial conclusion, though this kind of signaling was not implemented in this work, the proposed scheme is in principle settled just at the physical layer and does not require higher layer functionalities.

An alternative and more conventional approach would be a duplication of the photodetector and reflective modulator at the ONU, where a power splitter $\left(C_{S}\right)$ would also have to be placed in each wavelength branch as illustrated in Fig. 1(a) as "conventional ONU." This raises not only the cost but also introduces additional losses. Note that the introduction of the dual-operability requires the number of OLT receivers to be doubled compared to the transmission toward a single operator, regardless of the chosen ONU scheme.

\section{B. Dynamic Bandwidth Partitioning}

Once the detector can be operated as a remodulator, or vice versa, a configuration with two detectors or two remodulators is also feasible, allowing for fast and flexible electrical switching among downstream/upstream transmission configurations from $0 R_{b} / 2 R_{b}$ to $2 R_{b} / 0 R_{b}$, where $R_{b}$ is the nominal data rate for the data streams.

In the case of normal operation, corresponding to Fig. 2(a), the blue wavelength is remodulated at the ONU for upstream transmission. The detection and modulation bandwidth of the reflective modulators at the ONU will be in principle designed according to this data rate so that no upgrade path regarding a higher data rate per wavelength is provided. Alternatively to this normal operation mode, both wavelengths can be used for downstream detection (Fig. 2(b)) and upstream transmission (Fig. 2(c)), respectively. Although it is obvious that twice the data can be transmitted over two wavelengths, the added value of the proposed scheme is the flexible switch of the transmission direction: though just half-duplex transmission is achieved for this mode of unidirectional operation, no extra pair of transmitter and receiver is required as in [8] to change from the downstream to the upstream transmission direction or vice versa.

It is worth noting that each of the reflective modulators itself can also be operated in half-duplex transmission, allowing a variable upstream (or downstream) data rate between $0 R_{b}$ and $2 R_{b}$ (i.e., from 0 to $20 \mathrm{~Gb} / \mathrm{s}$ for the case that $R_{b}=10 \mathrm{~Gb} / \mathrm{s}$ ). In such a case there is still downstream (or upstream) operation except for the extreme cases of operating the ONU with twice the nominal data rate.

This second aspect of varying the data rate in downstream and upstream transmission can be advantageously used for the purpose of dynamic bandwidth partitioning. The switching between the rates of both data streams can be in principle performed rapidly, so that the data transmission can adapt itself to the actual bandwidth demands of the end-user application.

Regarding the complexity at the ONU, a similar comparison between the proposed and the conventional ONU counts, while at the OLT there is again the need for a doubling of receivers to obtain the dynamic bandwidth allocation functionality.

\section{BANDWIDTH CONSIDERATIONS}

The modulation bandwidth of SOAs depends typically on the current density in the active waveguide region and the optical input power. For the sake of completeness, the characteristics of the applied reflective modulators are presented in Fig. 3.

Figures 3(a) and 3(b) show the obtained electro-optical response for the commercial transistor-outline packaged RSOA (model Kamelian RSOA-18-TO-C) that was used in this work. While for bias currents around the transparency threshold just a low electro-optical bandwidth is guaranteed, values of 1.3 to $1.4 \mathrm{GHz}$ can be obtained for typical bias currents (Fig. 3(a)). The dependence on the optical input power is less pronounced for the used RSOA. For input power values from -7.5 to 

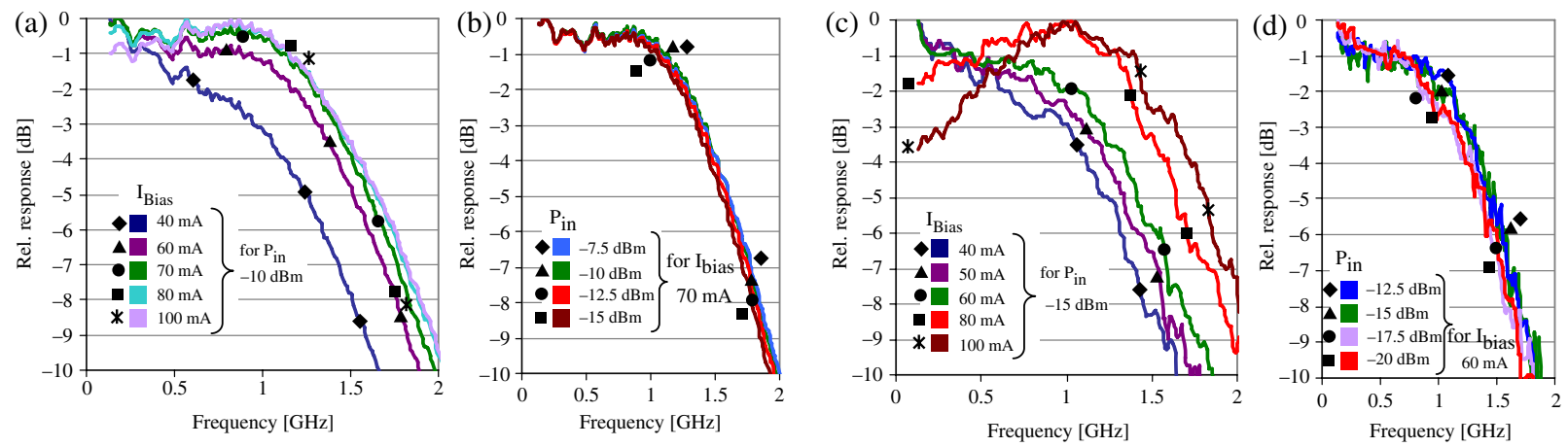

Fig. 3. (Color online) (a), (b) Measured electro-optical modulation bandwidth and (c), (d) opto-electrical detection bandwidth of the RSOA for different bias current settings and input power levels.

$-15 \mathrm{dBm}$, the electro-optical bandwidth stays around $1.4 \mathrm{GHz}$ for the chosen modulation bias current of $70 \mathrm{~mA}$ (Fig. 3(b)). Transmission at higher data rates of 5 and $10 \mathrm{~Gb} / \mathrm{s}$ has been demonstrated in conjunction with passive equalization [7,21]. The biasing condition for the RSOA current is then determined by a trade-off between a large modulation bandwidth and a high upstream extinction ratio (ER).

In the case of the more rarely found application of a RSOA as a detector, the detection bandwidth $B$ is limited by the carrier lifetime $\tau$. For a strong signal and negligible noise influence it can be found with

$$
B=\frac{1}{2 \pi \tau}, \quad \tau=\frac{1}{L} \int_{0}^{L}\left(\frac{d R(N)}{d N}+\frac{\Gamma}{W d} \frac{d g_{m}(z, N)}{d N} \Phi(z)\right)^{-1} d z,(1)
$$

where $L$ is the length of the SOA and $z$ is the coordinate inside the active waveguide in the propagation direction. $R$ is the recombination term that is determined by a series of recombination coefficients and depends on the carrier density $N$. $\Gamma$ and $W d$ are the confinement factor and the cross-section area of the active waveguide, respectively, $g_{m}$ is the material gain coefficient that depends indirectly on the bias current, and $\Phi$ is the photon flux due to an incident data signal. A more detailed analysis on the detection performance of a SOA can be found in [22].

Figures 3(c) and 3(d) present the detection bandwidth of the used RSOA for different conditions of input power and bias current. Similar to the case of modulation, the detection bandwidth shifts to higher values with higher values for the injected bias current (Fig. 3(c)) and the photon density (Fig. 3(d)) inside the active waveguide-as is also obvious from Eq. (1). The figures of merit that lead to the choice of the operational bias point are a high detection bandwidth and a high responsivity, the latter defined as the relation between the swing in the optical power of the input signal and the induced change in the bias current. Although a high bias current would guarantee a high detection bandwidth, the responsivity decreases when entering the saturation regime of the RSOA; since the signal and the spontaneous noise emission share the same carrier reservoir, a present modulation in the optical input signal will cause a reduced swing in the detected photocurrent when compared to operation in the more linear regime [23].
In contrast to bandwidth-limited devices such as RSOAs, EAMs offer a much higher modulation and detection bandwidth. Although this kind of modulator is still bound to carrier dynamics and is therefore slower than interferometric electrooptical devices, the short device length and, consequently, the low capacitance has allowed electro-optical bandwidths of $>50 \mathrm{GHz}$ to be achieved [24], especially with traveling-wave devices. Access applications with data rates of $10 \mathrm{~Gb} / \mathrm{s}$ can easily take advantage of these well-developed components without adding significant cost or bulky elements to the ONU [25].

\section{APPLICABILITY TO ACCESS Networks}

The feasibility of the proposed concept was assessed in terms of detection sensitivity and bandwidth requirements for the reflective modulators at the ONU. While the first will determine the compatible loss budget of the PON, the latter will have impact on the cost of the ONU. Two different kinds of reflective modulators were considered in this work: a commercially available RSOA and a SOA/REAM research prototype, which are suitable for low and high data rate transmission at $<2.5 \mathrm{~Gb} / \mathrm{s}$ and up to $10 \mathrm{~Gb} / \mathrm{s}$, respectively.

\section{A. RSOA as Reflective Modulator}

In a first step, the RSOA-based ONU was included in the WDM-PON shown in Fig. 4(a). The wavelength pair for the ONU consisted of optical carriers in the C-band at $1546.12 \mathrm{~nm}$ (R) and $1535.82 \mathrm{~nm}$ (B). Mach-Zehnder modulators (MZMs) were used at the OLT transmitter for imprinting the continuous-mode downstream with a high ER of $\sim 13 \mathrm{~dB}$. The wavelengths were then combined with an R/B coupler $\left(C_{T}\right)$ that was centered at $1544 \mathrm{~nm}$, and an erbium-doped fiber amplifier (EDFA) compensates the losses of the MZMs. Though this implementation looks costly at first glance, integrated transmitters have already been demonstrated [26].

The feeder and drop spans are composed of standard single-mode fibers (SMFs) of 25 and $6 \mathrm{~km}$ length. A $1 \times 40$ arrayed waveguide grating (AWG) with a $100 \mathrm{GHz}$ channel spacing acts as the signal distributing element of the 
(a)

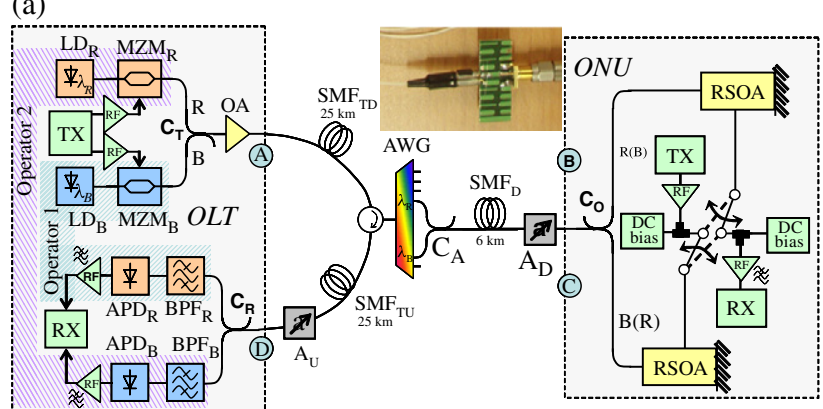

(b)

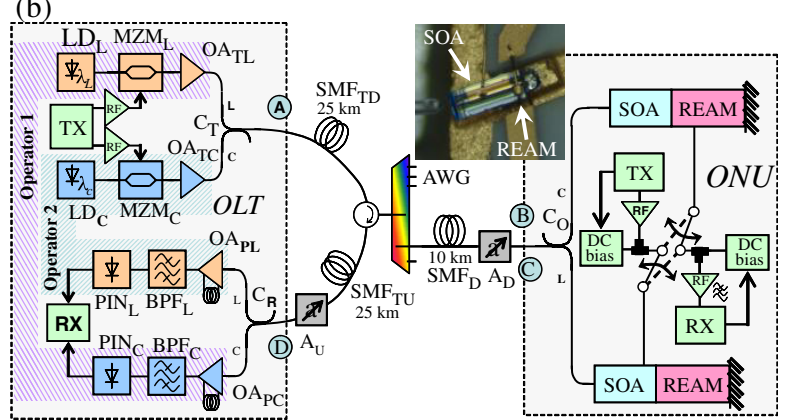

Fig. 4. (Color online) Experimental WDM-PON setup with (a) RSOA- and (b) SOA/REAM-based ONUs for the proof of the dual-operability and dynamic bandwidth allocation scheme.

WDM-PON. A red and a blue wavelength are recombined at each drop segment according to the dual-wavelength feed with the help of a further R/B coupler $\left(C_{A}\right)$, which could be avoided by an AWG with a specific free spectral range.

The OLT receiver included an $\mathrm{R} / \mathrm{B}$ separator to split the two wavebands and avalanche photodiodes (APDs) for the detection of the upstream. Optical bandpass filters (BPFs) enhance the rejection of the data channel in the opposite waveband as would also be the case in a realistic PON thanks to the WDM demultiplexer that is typically placed at the OLT receiver.

The RSOAs had a small signal gain of $21.7 \mathrm{~dB}$ with an optical $3 \mathrm{~dB}$ bandwidth of $54 \mathrm{~nm}$ that is centered at $1550 \mathrm{~nm}$ and a noise figure of $9 \mathrm{~dB}$. The modulating RSOA was biased at $68 \mathrm{~mA}$ and modulated with an $\mathrm{RF}$ current of $90 \mathrm{~mA}_{p p}$, resulting in an upstream ER of $8 \mathrm{~dB}$. The optimum RSOA bias for the detection of the downstream was found with $\sim 60 \mathrm{~mA}$, corresponding to an operation in the linear regime. The impact of the bandwidth limitation of the RSOA was particularly evaluated by transmission at a data rate of $2.5 \mathrm{~Gb} / \mathrm{s}$ in the simplest back-to-back case of the WDM-PON, in which several fibers were replaced by their equivalent attenuation.

The signal levels and optical signal-to-noise ratios (OSNRs) for the red (blue) wavelength were 6 (6) $\mathrm{dBm}$ and 44 (42.7) $\mathrm{dB}$ when being launched from the OLT booster (point A in Fig. 4(a)), $-8.1(-8.7) \mathrm{dBm}$ at the ONU input (B), 3.2 (1.6) $\mathrm{dBm}$ and 38.3 (37.8) $\mathrm{dB}$ after remodulation (C), and $-12.9(-14.9) \mathrm{dBm}$ at the input of the OLT receiver (D). This means a net gain of 11.3 (10.3) dB for the ONU. Degradation from Rayleigh backscattering (RB) at the drop fiber span did not cause any degradation due to the carrier-distributed scheme for the upstream transmission thanks to the high gain of the RSOA: the optical signal-to-RB ratio (OSRR) was $>40 \mathrm{~dB}$. On the other hand, a high ONU net gain can cause severe $\mathrm{RB}$ of the amplified reflected downstream into the incident downstream signal. However, it was proven that the OSRR was $>25 \mathrm{~dB}$ so that no significant performance degradation is derived.

The transmission performance was assessed with the help of bit error ratio (BER) measurements, which are shown in Fig. 5. Variable attenuators $\left(A_{D}, A_{U}\right)$ were therefore placed in front of the ONU and the OLT receiver.

The data rates for the simultaneously transmitted downstream and upstream were first chosen with $1.25 \mathrm{~Gb} / \mathrm{s}$. The downstream reception sensitivity in the back-to-back case is -17.8 (-11.6) $\mathrm{dBm}$ at the R- (B-) wavelength for a BER of $10^{-10}$ and a pseudorandom bit sequence (PRBS) of length $2^{7}-1$. This can fit to a WDM-PON scenario as will be discussed shortly. A penalty of 0.8 (1.1) $\mathrm{dB}$ is given for a PRBS of length $2^{31}-1$ due to the gain dynamics in the RSOA [27]. When the feeder and drop segments are composed of fiber, a penalty of $0.1(0.2) \mathrm{dB}$ is introduced, proving that transmission over fiber is not degraded by RB. The difference in the sensitivities between the red and blue bands is caused by the different rejection of the opposite waveband in the R/B splitter; the crosstalk from the red (upstream seed) into the blue (downstream) wavelength was $-23.4 \mathrm{~dB}$ while it was $15 \mathrm{~dB}$ lower for the opposite case of receiving the red wavelength.

For the upstream measurements the input power of the ONU was fixed to the delivered one, according to the losses in the PON. The penalty in the back-to-back case between the two PRBS lengths is $0.4(0.9) \mathrm{dB}$ at a BER of $10^{-10}$ Crosstalk between the red and blue upstream wavelengths and, consequently, a difference in the reception sensitivity is prevented thanks to the additional narrowband optical bandpass filter used at the OLT receiver. When transmitting over fiber spans, the penalty related to the back-to-back case is $0.4(0.2) \mathrm{dB}$.

The power margin for the downstream, defined between the delivered optical power and the reception sensitivity, is 7.6 (1.5) $\mathrm{dB}$ and 17 (15.4) $\mathrm{dB}$ for the upstream.

Full-duplex operation at $2.5 \mathrm{~Gb} / \mathrm{s}$ suffers from a reduced detection and modulation response of the RSOAs, which introduces error floors in the downstream reception. However, considering a Reed-Solomon $(255,239)$ forward error correction (FEC) as is proposed in next-generation PON standards [28], transmission is possible at a BER level of $10^{-4}$ with a penalty of 2.9 (2.9) $\mathrm{dB}$ for the downstream, while the upstream suffers from a degradation of $3.1(2.8) \mathrm{dB}$ at a BER of $10^{-10}$.

The compatible optical loss budget for the PON with a nominal data rate of $1.25 \mathrm{~Gb} / \mathrm{s}$ is limited by the downstream reception to 21.7 (16.2) dB without FEC and to 29.5 (24.2) $\mathrm{dB}$ with FEC. It shall be recalled that the penalty for the blue wavelength could be easily avoided by an R/B filter with better rejection at the ONU. 

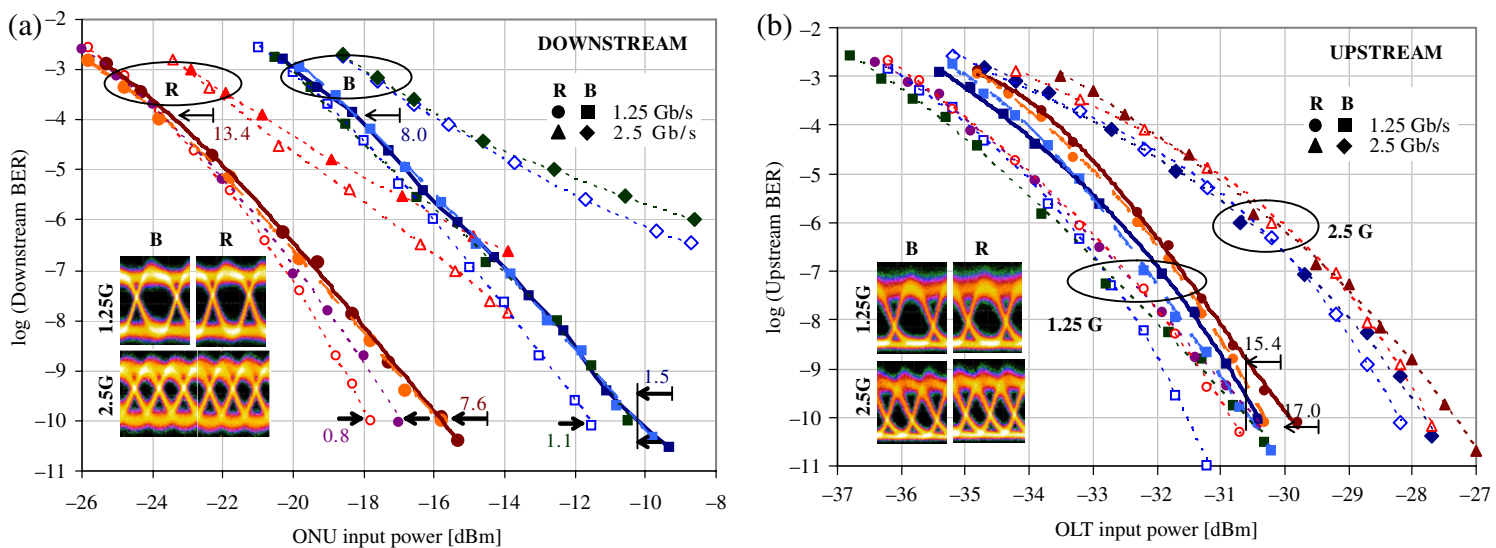

Fig. 5. (Color online) BER measurements for (a) downstream and (b) upstream. Dotted curves indicate the back-to-back case, dashed curves indicate the presence of a feeder but no drop fiber, and solid curves indicate present feeder and drop fibers. Hollow markers show results for a PRBS of length $2^{7}-1$, while filled markers represent a PRBS of length of $2^{31}-1$. Single ended arrows indicate power margins for the reception according to the delivered optical power.

\section{B. SOA/REAM as a Reflective Modulator}

Higher data rates can be achieved when the RSOAs in the ONU are substituted by suitable modulators with higher electro-optical bandwidth. A promising candidate is the SOA/REAM, whose EAM section can be used as a fast PIN diode [19]. To emulate the burst-like data delivery as is the case for dynamic bandwidth partitioning, burst-mode operation was investigated in addition.

An ONU that was modified according to a SOA/REAM-based downstream detector and upstream modulator was evaluated in a similar WDM-PON (Fig. 4(b)) as was used in the previous section. The SOA/REAM chip had a $70 \mu \mathrm{m}$ long EAM with a high-reflective coating on its rear facet and a $400 \mu \mathrm{m}$ long SOA section and a passive waveguide of $150 \mu \mathrm{m}$ length with an anti-reflective coating on its front facet. Since the SOA/REAM was optimized for the lower L-band [29], the red/blue allocation was altered toward a C/L-waveband scheme that employs wavelengths at 1560.61 and $1592.95 \mathrm{~nm}$. The spacing between these optical carriers fits to the free spectral range of the $1 \times 8$ AWG that was $32.5 \mathrm{~nm}$.

Note that the APD-based receiver at the OLT was replaced by a combination of EDFA and PIN diode since no $10 \mathrm{~Gb} / \mathrm{s}$ burst-mode capable APD was available. A dispersioncompensating fiber (DCF) with a dispersion of $-671 \mathrm{ps} / \mathrm{nm}$ was included in the OLT receiver to reduce the interplay of the EAM chirp with the SMF for the $10 \mathrm{~Gb} / \mathrm{s}$ upstream signal.

Both data streams were transmitted in continuous mode as well as in burst mode to further validate the concept of dynamic bandwidth partitioning. For this reason a burst-mode transmitter at the OLT and the ONU was generating a $125 \mu \mathrm{s}$ gigabit PON-compatible frame (with a $60 \mathrm{~ns}$ preamble in the case of the downstream) and duty cycles of $1: 4,1: 2$, and $1: 1$ (i.e., continuous mode). There was no specific data framing for the generated burst. Accordingly, the data packet contained mostly payload data besides the preamble. The received data packets for downstream and upstream at the ONU and OLT, respectively, are shown in Fig. 6.
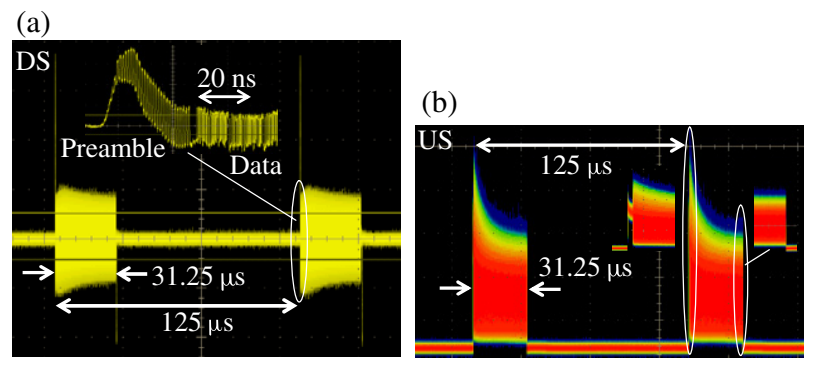

Fig. 6. (Color online) Burst-mode traffic with a duty cycle of 1:4 for (a) the downstream and (b) the upstream transmission.

Since there was only one chip available, the reception and transmission was not performed concurrently. However, no crosstalk into the channel of the other waveband has been observed when emulating the upstream signal with a laser diode at the ONU, thanks to the good rejection of the opposite waveband at the $\mathrm{C} / \mathrm{L}$ splitter.

The SOA section of the downstream receiver was gated with a bias current of $120 \mathrm{~mA}$, according to the chosen duty cycle of the transmitted burst-mode downstream. The EAM section was biased highly negative to act as a detector. The bias voltages were $-1.8 \mathrm{~V}(-4.2 \mathrm{~V})$ at the $\mathrm{C}$ - (L-) band wavelength. The C-band EAM bias was thereby lower due to the closer detuning from the absorption edge.

For upstream transmission the EAM section was biased at $-1.1 \mathrm{~V}(-1.6 \mathrm{~V})$ and modulated with $2 \mathrm{~V}_{p p}\left(3 \mathrm{~V}_{p p}\right)$, respectively, while the SOA was gated as mentioned for the downstream reception. The L-band wavelength was changed to $1585.36 \mathrm{~nm}$ for the upstream for a better alignment with available L-band filters at the optical OLT receiver. Upstream ERs of 9.3 and $8.1 \mathrm{~dB}$ were obtained for the $\mathrm{C}$ - and L-band wavelength at the output of the ONU.

The signal levels and OSNRs for the C- (L-) band wavelength were $7(7) \mathrm{dBm}$ and $42.3(43.1) \mathrm{dB}$ after the OLT booster (point A in Fig. 4(b)) and $-4.2(-4.6) \mathrm{dBm}$ at the ONU input (B). The ONU input was fixed to $-7 \mathrm{dBm}$ for the upstream 

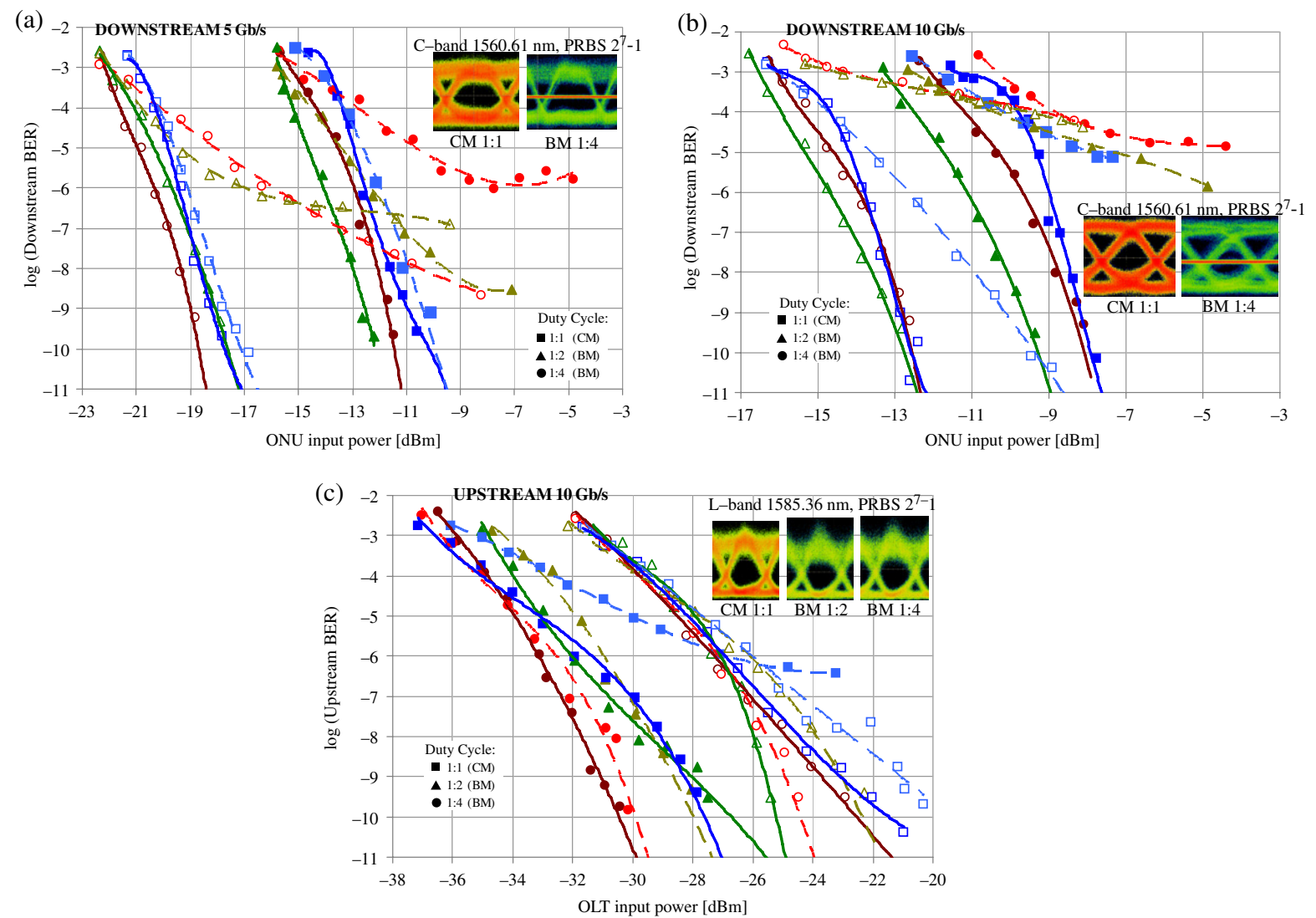

Fig. 7. (Color online) BER measurements for (a) $5 \mathrm{~Gb} / \mathrm{s}$ downstream, (b) $10 \mathrm{~Gb} / \mathrm{s}$ downstream, and (c) $10 \mathrm{~Gb} / \mathrm{s}$ upstream in the C-band (filled markers) and the L-band (blank markers) in continuous mode (CM) and burst mode (BM). Solid and dashed lines represent a PRBS of length $2^{7}-1$ and $2^{31}-1$, respectively. The horizontal line in the burst-mode eye diagram of the downstream stems from the AC-coupled off-level.

measurements so that an error-free downstream reception can be guaranteed, as will be shown shortly. The further power levels and OSNRs are $-2.0(-0.5) \mathrm{dBm}$ and $36.6(39.3) \mathrm{dB}$ after remodulation at the ONU output (C) and -13.6 (-12.5) $\mathrm{dBm}$ at the input of the OLT receiver (D).

The downstream in the L-band, whose BER measurements are presented in Figs. 7(a) and 7(b) for data rates of 5 and $10 \mathrm{~Gb} / \mathrm{s}$, respectively, benefits from the improved L-band response of the SOA/REAM chips by 3.5 to $6 \mathrm{~dB}$ when compared to the $\mathrm{C}$-band transmission. The latter reaches sensitivities below -9.5 (at $5 \mathrm{~Gb} / \mathrm{s}$ ) and $-7.5 \mathrm{dBm}($ at $10 \mathrm{~Gb} / \mathrm{s}$ ) for a PRBS of length $2^{7}-1$ at a BER of $10^{-10}$ or a PRBS of length $2^{31}-1$ at the FEC threshold. Note that the $60 \mathrm{~ns}$ long preamble of the downstream, shown in Fig. 6(a) and excluded from the BER, is distorted due to the AC-coupling of the electronic receiver at the ONU.

The C-band signal benefits from a 3 to $5 \mathrm{~dB}$ better sensitivity for the $10 \mathrm{~Gb} / \mathrm{s}$ upstream reception since the used C-band EDFA at the OLT preamplifier provided a higher gain than the slightly worse performing L-band EDFA. Sensitivities below $-22 \mathrm{dBm}$ were obtained for the L-band at a BER of $10^{-10}$, while reception at the FEC level is possible with $-29 \mathrm{dBm}$ (Fig. 7(c)). Since the EDFA was operated without gain stabilization, gain transients were present for the burst-mode upstream. However, with a short packet length the signal experiences excess gain (see Fig. 6(b)) while no severe asymmetry is visible in the corresponding eye diagram (Fig. 7(c)). For this reason, the sensitivity of the burst-mode upstream is slightly improved compared to its continuous mode. No strong differences between the different PRBS lengths have been observed compared to the case of downstream reception, since the incident unmodulated optical carrier is partially clamping the carrier density inside the SOA.

Similar to the RSOA-based ONU, the loss budget of the PON is limited by the downstream reception to $16(16.5) \mathrm{dB}$ for the C- (L-) band wavelength but can in principle be increased with a better fiber-to-waveguide coupling, e.g., with the help of an integrated mode size converter.

\section{CONCLUSion}

An ONU upgrade at the physical layer, featuring dualoperability and dynamic bandwidth provisioning, has been demonstrated for a carrier-distributed WDM-PON with reflective modulators at the colorless customer premises equipment. The novel ONU design substitutes the conventional photodiode-based receiver by a more functional photonic device for the flexible modulation/detection function, while the small form factor of the ONU is kept. 
The validation of $10 \mathrm{~Gb} / \mathrm{s}$ downstream and upstream operation in burst mode shows the feasibility of bandwidth partitioning up to $20 \mathrm{~Gb} / \mathrm{s}$ with the proposed tandemmodulator configuration, though the reception sensitivity for the downstream is still subject to improvement to obtain higher compatible optical loss budgets for the access network.

\section{ACKNOWLEDGMENTS}

This work was supported in part by the European FP7 EUROFOS and SARDANA projects, the French ANR-ANTARES, and the Spanish MICINN TEC2008-01887 project and FPU program.

\section{REFERENCES}

[1] Y. Xiao, X. Du, J. Zhang, F. Hu, and S. Guizani, "Internet protocol television (IPTV): The killer application for the next-generation Internet," IEEE Commun. Mag., vol. 45, no. 11, pp. 126-134, Nov. 2007.

[2] H. Shinohara, "FTTH experiences in Japan," J. Opt. Netw., vol. 6, no. 6, pp. 616-623, June 2007.

[3] F. J. Effenberger, K. McCammon, and V. O'Byrne, "Passive optical network deployment in North America," J. Opt. Netw., vol. 6, no. 7, pp. 808-818, July 2007.

[4] A. Sugita, A. Kaneko, K. Okamoto, M. Itoh, A. Himeno, and Y. Ohmori, "Very low insertion loss arrayed-waveguide grating with vertically tapered waveguides," IEEE Photon. Technol. Lett., vol. 12 , no. 9, pp. 1180-1182, Sept. 2000.

[5] S. J. Park, C. H. Lee, K. T. Jeong, H. J. Park, J. G. Ahn, and K. H. Song, "Fiber-to-the-home services based on wavelengthdivision-multiplexing passive optical network," J. Lightwave Technol., vol. 22, no. 11, pp. 2582-2591, Nov. 2004.

[6] Q. T. Nguyen, L. Bramerie, P. Besnard, A. Shen, A. Garreau, C. Kazmierski, G. H. Duan, and J. C. Simon, "24 channels colorless WDM-PON with L-band $10 \mathrm{~Gb} / \mathrm{s}$ downstream and C-band $2.5 \mathrm{~Gb} / \mathrm{s}$ upstream using multiple-wavelengths seeding sources based on mode-locked lasers," in Optical Fiber Communication Conf., San Diego, CA, Mar. 2010, OThG6.

[7] B. Schrenk, G. de Valicourt, M. Omella, J. A. Lazaro, R. Brenot, and J. Prat, "Direct $10 \mathrm{~Gb} / \mathrm{s}$ modulation of a single-section RSOA in PONs with high optical budget," IEEE Photon. Technol. Lett., vol. 22, no. 6, pp. 392-394, Mar. 2010.

[8] C. W. Chow, L. Xu, C. H. Yeh, H. K. Tsang, W. Hofmann, and M. C. Amann, "40-Gb/s upstream transmitters using directly modulated 1.55- $\mu \mathrm{m}$ VCSEL array for high-split-ratio PONs," IEEE Photon. Technol. Lett., vol. 22, no. 5, pp. 347-349, Mar. 2010.

[9] M. Forzati and C. P. Larsen, "On the symmetry requirements for tomorrow's fibre access networks," in 11th Int. Conf. on Transparent Optical Networks, Ponta Delgada, Portugal, June 2009, Tu.B5.3.

[10] J. R. Stern, J. W. Balance, D. W. Faulkner, S. Hornung, D. Payne, and K. Oakley, "Passive optical local networks for telephony applications and beyond," Electron. Lett., vol. 23, no. 24, pp. 1255-1256, Nov. 1987.

[11] C. Bock, J. Prat, and S. Walker, "Hybrid WDM/TDM PON using the AWG FSR and featuring centralized light generation and dynamic bandwidth allocation," J. Lightwave Technol., vol. 23, no. 12, pp. 3981-3988, Dec. 2005.

[12] D. Qian, J. Hu, P. N. Ji, T. Wang, and M. Cvijetic, "10-Gb/s OFDMA-PON for delivery of heterogeneous services," in Optical Fiber Communication Conf. and Expo. and the Nat. Fiber Optic Engineers Conf., San Diego, CA, Feb. 2008, OWH4.
[13] C. W. Chow, C. H. Yeh, C. H. Wang, C. L. Wu, S. Chi, and C. Lin, "Studies of OFDM signal for broadband optical access networks," IEEE J. Sel. Areas Commun., vol. 28, no. 6, pp. 800-807, Aug. 2010.

[14] A. Barbieri, G. Colavolpe, T. Foggi, E. Forestieri, and G. Prati, "OFDM versus single-carrier transmission for $100 \mathrm{Gbps}$ optical communication," J. Lightwave Technol., vol. 28, no. 17, pp. 2537-2551, Sept. 2010.

[15] P. Chanclou, S. Gosselin, J. Fernandez, V. Lopez, and E. Zouganeli, "Overview of the optical broadband access evolution: A joint article by operators in the IST network of excellence e-Photon/One," IEEE Commun. Mag., vol. 44, no. 8, pp. 29-35, Aug. 2006

[16] J. Prat, C. Arellano, V. Polo, and C. Bock, "Optical network unit based on a bidirectional reflective semiconductor optical amplifier for fiber-to-the-home networks," IEEE Photon. Technol. Lett., vol. 17, no. 1, pp. 250-252, Jan. 2005.

[17] E. Udvary and T. Berceli, "Semiconductor optical amplifier for detection function in radio over fiber systems," J. Lightwave Technol., vol. 26, no. 15, pp. 2563-2570, Aug. 2008.

[18] B. Schrenk, J. A. Lazaro, V. Polo, and J. Prat, "Multi-operability in WDM-PONs with electrically reconfigurable RSOA-based optical network units," in Access Networks and In-house Communications, Karlsruhe, Germany, June 2010, ATuB4.

[19] J. Bauwelinck, B. Schrenk, C. Kazmierski, J. A. Lazaro, J. Prat, and X. Z. Qiu, "Multi-operability and dynamic bandwidth allocation in PONs with electrically reconfigurable SOA/REAM-based ONUs," in 36th European Conf. and Exhibition on Optical Communication, Torino, Italy, Sept. 2010, Th.10.B.4.

[20] A. Garreau, C. Kazmierski, D. Chiaroni, M. Le Pallec, J. Decobert, J. Landreau, D. Carpentier, and J. G. Provost, "10 Gbit/s drop and continue colorless operation of a $1.5 \mu \mathrm{m}$ AlGaInAs reflective amplified electroabsorption modulator," in European Conf. on Optical Communication, Cannes, France, Sept. 2006, We.1.6.5.

[21] P. Chanclou, F. Payoux, T. Soret, N. Genay, R. Brenot, F. Blache, M. Goix, J. Landreau, O. Legouezigou, and F. Mallécot, "Demonstration of RSOA-based remote modulation at 2.5 and $5 \mathrm{Gbit} / \mathrm{s}$ for WDM PON," in Optical Fiber Communication Conf. and Expo. and the Nat. Fiber Optic Engineers Conf., Anaheim, CA, Mar. 2007, OWD1.

[22] M. J. Connelly and R. F. O'Dowd, "Travelling-wave semiconductor laser amplifier detector noise characteristics," IEE Proc.: Optoelectron., vol. 142, no. 1, pp. 23-28, Feb. 1995.

[23] M. Gustavsson, A. Karlsson, and L. Thylen, "Traveling wave semiconductor laser amplifier detectors," J. Lightwave Technol. vol. 8, no. 4, pp. 610-617, Apr. 1990.

[24] C. Kazmierski, A. Konczykowska, F. Jorge, F. Blache, M. Riet, C. Jany, and A. Scavennec, " $100 \mathrm{~Gb} / \mathrm{s}$ operation of an AlGaInAs semi-insulating buried heterojunction EML," in Optical Fiber Communication Conf., San Diego, CA, Mar. 2009, OThT7.

[25] C. Kazmierski, "Advances in remote amplified modulator developments for applications from $10 \mathrm{~Gb} / \mathrm{s}$ WDM access to $100 \mathrm{~Gb} / \mathrm{s}$ core networks," in 36th European Conf. and Exhibition on Optical Communication, Torino, Italy, Sept. 2010, Mo.1.F.1

[26] M. Kato, R. Nagarajan, J. Pleumeekers, P. Evans, A. Chen, A. Mathur, A. Dentai, S. Hurtt, D. Lambert, P. Chavarkar, M. Missey, J. Bäck, R. Muthiah, S. Murthy, R. Salvatore, C. Joyner, J. Rossi, R. Schneider, M. Ziari, F. Kish, and D. Welch, "40-channel transmitter and receiver photonic integrated circuits operating at per channel data rate $12.5 \mathrm{Gbit} / \mathrm{s}$, Electron. Lett., vol. 43, no. 8, pp. 468-469, Apr. 2007.

[27] X. Wei, Y. Su, X. Liu, J. Leuthold, and S. Chandrasekhar, "10-Gb/s RZ-DPSK transmitter using a saturated SOA as a power booster and limiting amplifier," IEEE Photon. Technol. Lett., vol. 16, no. 6, pp. 1582-1584, June 2004. 
[28] "10-gigabit capable passive optical networks (XG-PON)," ITU-T Recommendation G.987, 2010.

[29] N. Dupuis, J. Décobert, C. Jany, F. Alexandre, A. Garreau, N. Lagay, F. Martin, D. Carpentier, J. Landreau, and C. Kazmierski, "10-Gb/s AlGaInAs colorless remote amplified modulator by selective area growth for wavelength agnostic networks," IEEE Photon. Technol. Lett., vol. 20, no. 21, pp. 1808-1810, Nov. 2008.

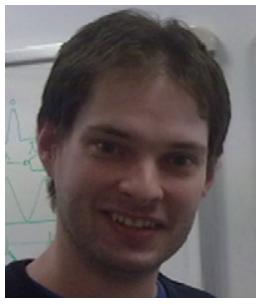

Bernhard Schrenk was born in 1982 in Austria and received the B.Sc. ('05) and M.Sc. ('07) degrees in microelectronics from the Technical University of Vienna. From 2007 to early 2011 he obtained his Ph.D. degree at Universitat Politècnica de Catalunya (UPC) BarcelonaTech, Spain. His Ph.D. thesis focused on multifunctional optical network units for next-generation fiber-to-the-home access networks.

He was at the Institute of Experimental Physics of Prof. A. Zeilinger, where he was involved in the realization of a first commercial prototype for a quantum cryptography system, within the European SECOQC project. Currently, he is a Post-doctoral Researcher at the National Technical University of Athens, Greece. His research interests include next-generation passive optical fiber-to-the-home networks for long reach and high data rate. Dr. Schrenk was engaged in several European projects such as SARDANA, BONE, BOOM, APACHE, GALACTICO, and EURO-FOS.

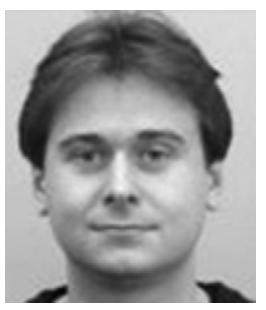

Johan Bauwelinck was born in Sint-Niklaas, Belgium, in 1977. He received the Engineering degree in applied electronics and the Ph.D. degree in applied sciences, electronics, from Ghent University, Ghent, Belgium, in 2000 and 2005, respectively. He has been a Research Assistant in the INTEC_design Laboratory, Ghent University, since 2000, and he is currently a full-time tenure track Professor. His research is focused on high-speed, high-frequency (opto-) electronic circuits and systems and their applications on the chip and board level, including optical access networks, automotive optical networks, and RF design for wireless communication and ranging.

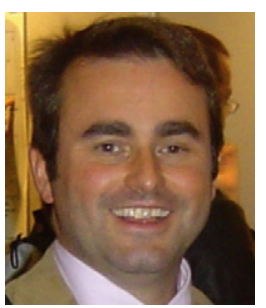

José A. Lázaro received the M.Sc. degree in physics in 1993 and the $\mathrm{Ph} . \mathrm{D}$. degree from the University of Zaragoza (Spain) in 1999 for his research work on erbium-doped waveguide amplifiers (EDWAs). He is currently a Ramon-y-Cajal Researcher at the Optical Communications Group of the Department of Signal Theory and Communications of the Universitat Politècnica de Catalunya (UPC), teaching at the ETSETB Engineering School of UPC in Barcelona, and the Master in Photonics "PhotonicsBCN." Prior to this position, he was a Senior Researcher at the Optical Transmission and Broadband Technologies Laboratory of the Aragon
Institute for Engineering Research (I3A) in Huesca, Spain, and an R\&D Engineer at ALCATEL in Stuttgart, Germany, in the Department of Passive Optical Components (2000-2002) and in the Department of Optical Transmission Systems (2002-2004). He has been participating in several European projects (e-Photon/One, BONE, EURO-FOS, and SARDANA) on optical transmission, monitoring, subsystems, and access networks.

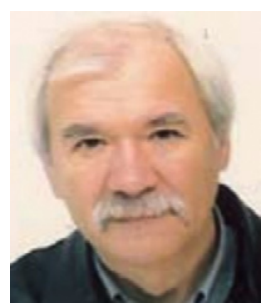

Christophe Kazmierski is currently with the Alcatel-Thales III-V Lab, Marcoussis, France, where he is involved in research on $40-400 \mathrm{~Gb} / \mathrm{s}$ electro-absorption modulatorbased sources.

Christophe Kazmierski contributed to pioneering work on long-wavelength lasers at the Central Research Laboratory of Thomson-CSF. $\mathrm{He}$ also headed the Laser Department of France Telecom R\&D, working on laser-based photonic circuits. At the III-V Laboratory, he is focused on 100-400 Gbps electro-absorption modulator based photonic integrated sources. $\mathrm{He}$ has authored over 300 papers and 18 patents in the field of III-V semiconductors. He has also served on program committees at International Semiconductor Laser, Microwave Photonics and Indium Phosphide and Related Materials conferences. He has been involved in a dozen cooperative international programs including ESPRIT263, RACE I, RACE II, EUREKA, IST, and National RNRT and ANR programs.

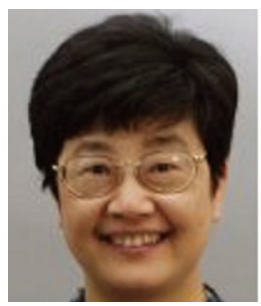

Xing-Zhi Qiu received a Ph.D. degree in electronics engineering from Ghent University, Belgium, in 1993. Since 1986 she has been with the INTEC_design Laboratory of Ghent University and gained 24 years of $R \& D$ experience in the design of high-speed opto-electronic subsystems and physical layer demonstrators. She co-authored more than 110 publications in the field of advanced telecom systems, broadband access networks, and mixed-mode chip development and 5 patents on communications.

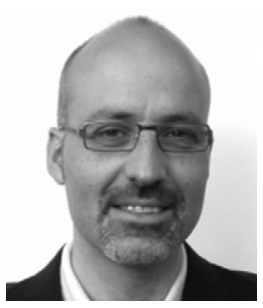

Josep Prat received the M.S. degree in telecommunications engineering in 1987 and the $\mathrm{Ph} . \mathrm{D}$. degree from the Universitat Politècnica de Catalunya (UPC), Barcelona, in 1995.

$\mathrm{He}$ is a Full Professor in the Optical Communications Group of the Signal Theory and Communications Department of the UPC. He has mainly investigated broadband optical communications with emphasis on high-bitrate optical systems, access networks, and WDM transmission design and impairment control. He has participated in several European projects (Euro-Fos, BONE, e-Photon/One, LION, MEPHISTO, MOON, SONATA, and RACE1027) on optical transport and access networks and leads the FP7 SARDANA project (www.ict-sardana.eu) on next-generation FTTH networks. In 1998 he was a guest scientist at the University College of London. $\mathrm{He}$ is the editor of the books Fiber-to-the-Home Technologies and Next-Generation FTTH Networks. 